Article

\title{
A Thermodynamic Entropy Approach to Reliability Assessment with Applications to Corrosion Fatigue
}

\author{
Anahita Imanian * and Mohammad Modarres \\ Center for Risk and Reliability, Department of Mechanical Engineering, University of Maryland, \\ College Park, MD 20742, USA; E-Mail: modarres@umd.edu \\ * Author to whom correspondence should be addressed; E-Mail: aimanian@umd.edu. \\ Academic Editors: Morin Celine, Bernard Desmet, and Fethi Aloui
}

Received: 31 August 2015 / Accepted: 10 October 2015 / Published: 16 October 2015

\begin{abstract}
This paper outlines a science-based explanation of damage and reliability of critical components and structures within the second law of thermodynamics. The approach relies on the fundamentals of irreversible thermodynamics, specifically the concept of entropy generation as an index of degradation and damage in materials. All damage mechanisms share a common feature, namely energy dissipation. Dissipation, a fundamental measure for irreversibility in a thermodynamic treatment of non-equilibrium processes, is quantified by entropy generation. An entropic-based damage approach to reliability and integrity characterization is presented and supported by experimental validation. Using this theorem, which relates entropy generation to dissipative phenomena, the corrosion fatigue entropy generation function is derived, evaluated, and employed for structural integrity and reliability assessment of aluminum 7075-T651 specimens.
\end{abstract}

Keywords: irreversible thermodynamics; entropy generation; reliability and structural integrity; corrosion fatigue

\section{Introduction}

Data-driven stochastic and probabilistic-based methods that underlie reliability prediction and structural integrity assessment methods have remained unchanged for decades, and involve considerable uncertainty and conservatism. Consequently, a more realistic prediction of the performance of structural material degradation requires considerable fundamental research. In fact, there is a substantial gap 
between available reliability and integrity techniques and the science of materials degradation dynamics used to model early failure prediction, formally develop accelerated failure testing, and predict the Remaining Useful Life (RUL) of critical components and structures. This paper reports an alternative approach for damage assessment based on the fundamentals of thermodynamics, from which the reliability of components and integrity of structures was derived. Subsequent experimental results validate the proposed approach as a corrosion fatigue degradation mechanism.

In contrast with the first law of thermodynamics, which establishes the equivalence between heat and work but is silent on the limitations of the transformation of one into the other, the second law of thermodynamics defines such limitations and illustrates that natural processes evolve spontaneously in one direction only through the increase of entropy [1]. An interesting aspect of the principle of entropy increase is the special light it sheds on the concept of time direction and evolution of events. In accordance with the principle of entropy increase, manufactured organized components return to their natural conditions through degradation, which reduces the integrity of material properties to the point where the components are no longer functional [2].

To characterize the reduction of integrity, "damage" is a common term that is widely used in various disciplines [3]. The concept of damage is somewhat abstract, and its definition relies on the field variables used as markers of damage to describe the anticipated aging or degradation process [4]. Differences in these variables are the reasons that several definitions and measures of damage exist. For example, fatigue damage has been described in several ways: reduction in the Young's modulus; cumulative number of cycles ratio; reduction of load-carrying capacity; crack length; and energy dissipation. Other markers of damage include surface deterioration or volumetric deterioration of material. As such, fatigue and creep are processes that damage the volume elements of a structure, while corrosion and wear cause surface damage. Moreover, the definition of damage varies at different geometric scales. For example, in the corrosion fatigue degradation mechanism, transmission electron microscopy and dislocation modeling studies show that at the nano-scale adsorbed hydrogen ions localize plastic deformation and lower the energy required for fracture, resulting in degradation [5]. At the micro-scale level, it has been shown that weakness of oxide film for extensive plastic deformation relative to the underlying metal may result in the localization of slip bands and a reduction in nearsurface plasticity, ultimately leading to the formation of dislocation structures and voids [6]. At the meso-scale level, damage constitutes the growth and coalescence of micro-cracks that initiate a crack. At a macro-scale level, damage is defined as the growth and propagation of macro-cracks that result in a final fracture [7]. Therefore, a more objective, consistent, and broad definition of damage is necessary and plausible. To attain this goal, this paper elaborates on the concept of material damage within the thermodynamic framework.

In terms of thermodynamics, all forms of damage share a common characteristic: dissipation of energy. In thermodynamics, dissipation of energy (in processes such as chemical reactions, release of heat, diffusion of materials, and plastic deformation) is the basic measure of irreversibility and the main feature of degradation processes in material $[2,8]$. In turn, dissipation of energy can be quantified by the entropy generation within the context of irreversible thermodynamics [9-11]. Therefore, dissipation (or its equivalent, entropy generation) can be considered as a substitute for characterization of damage. This forms the basis of the theory pursued in this study, in which entropy generation is considered as a 
broad index of damage and thus offers a science-based alternative for modeling a wide range of damage processes and describes a more fundamental science of reliability and integrity assessment.

Although the concept of entropy generation for the study of dissipative processes is not a new approach and has a rich history of research in chemistry, material science, and mechanics, its application to practical problems has been realized only in recent years. A comprehensive review of applications of entropy concepts in various research areas is given by Martyushev and Seleznev and Amiri and Modarres [2,12]. Entropy can be used to quantify the behavior of irreversible degradation, including plasticity and dislocations [13,14], erosion-corrosion [15], wear and fracture [16-20], fatigue [21-26], fretting corrosion [27], thermal degradation [28], and associated failure of tribological components $[29,30]$. The benefit of employing entropy generation to characterize materials damage is that entropy generation can provide a unified measure of damage, allowing for incorporation of multiple competing and common-cause degradation mechanisms that can be explicitly expressed in terms of physically measurable quantities. Furthermore, entropy as a thermodynamic state function is independent of the path of the failure (which commonly depends on factors such as environmental condition, geometry, load, and frequency of load) from the initial state to the final failed state of the material, considering a known endurance limit [2,21].

The assessment of the corrosion fatigue degradation mechanism as an example of the proposed entropic-based reliability and integrity assessment is of technical and economic interest, as it can severely jeopardize the structural integrity and safety of critical structures such as off-shore structures, naval vessels, and naval airframes [31-33]. Common practices for corrosion fatigue structural integrity assessment in metals and alloys have isolated and evaluated the damage characteristics at different life stages. As such, the corrosion fatigue damage process is divided into four regimes: the pit nucleation step, the nucleated pit to grow stage, surface crack initiation and growth into a through-crack phase, and through-crack growth to a prescribed critical length step. For each of these stages, the literature offers empirical models for life estimation of metal and alloys [34-41]. In these models, the pit growth rate typically follows the Kondo model [34], whereby the pit remains hemispherical in shape and grows at a constant volumetric rate. The Linear Elastic Fracture Mechanism (LEFM) and Elasto-Plastic Fracture Mechanism (EPFM) are often applied at the crack propagation stage and final instability $[37,42,43]$. The lifetime of a component subject to corrosion fatigue is estimated by the sum of the lifetime obtained from empirical models developed for each stage. In considering the use of entropy for corrosion fatigue structural integrity assessment, the aims of this paper are threefold. First, we seek to provide practical support for the use of entropy generation as a damage index derived in terms of multiple dissipative processes occurring in corrosion fatigue. Second, we explore the hypothesis that fracture entropies are almost constant, independent of the loading condition at the failure stage. Finally, we evaluate the advantages of using an entropy damage index for assessing structural integrity.

\section{Entropy Generation and Damage}

It was stated earlier that the damage resulting from the degradation processes could be viewed as the consequence of a dissipation of energies (see Figure 1) that can be more generally expressed by entropy as its index such that: 


\section{Damage $\equiv$ Entropy}

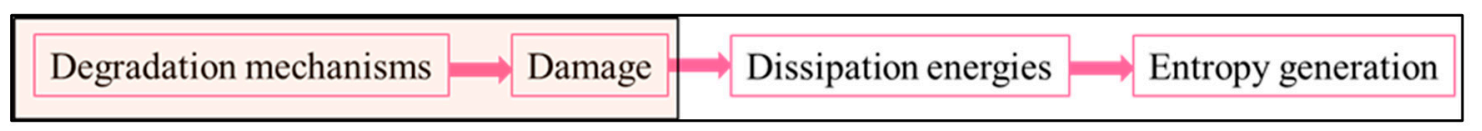

Figure 1. Entropy generation's relationship to damage.

An immediate question that arises is how to characterize the deterioration of integrity due to the resulting damage in terms of the entropy generation. Consistent with the second law of thermodynamics, entropy does not obey the conservation law. Processes occurring inside the system may be reversible or irreversible. Reversible processes inside a system may lead to transfer of entropy, resulting from heat and material exchange from one part of the system to other parts of the interior, but these processes do not generate entropy. Irreversible processes inside a system, however, do generate entropy, and hence this factor must be taken into account in computing the level of entropy.

\section{Fundamentals of Irreversible Thermodynamics}

For a system with properties being continuous functions of space and time, irreversible processes can be described by formulating the first and second laws of thermodynamics. If the system involves chemical reaction and work of any forms, Gibbs [44] shows that the change in internal energy, $U$, of the system on the basis of combining the first and second laws of thermodynamics would be:

$$
\dot{U}=T \dot{S}+\dot{W}+\sum_{i=1}^{m} X_{i} J_{i}+\sum_{k=1}^{r} \mu_{k} v_{k},
$$

where $S$ is the entropy of the system, $T$ is temperature, $W$ is the mechanical work due to deformation in in the bulk of the system, $X_{i}$ is generalized thermodynamic force (such as pressure), $J_{i}$ is the generalized generalized thermodynamic flux (such as volume rate), $\mu_{k}$ is the chemical potential, and $v_{k}$ is the chemical reaction rate of the $k$-th component.

Note that $\dot{W}$ in Equation (1) is associated with the work of quasi-conservative forces and $X_{i} J_{i}$ is associated with the work of dissipative forces. According to Ziggler [45], forces applied on the system can be divided into quasi-conservative and dissipative forces. Quasi-conservative forces can be derived from free energy and do not contribute to dissipation [2].According to the principles of thermodynamics for any macroscopic system, the variation of the entropy, $S$, of the system can be written as the sum of two terms:

$$
d S=d^{e} S+d^{d} S,
$$

where superscripts $e$ and $d$ represent the exchange and dissipative parts of the entropy, respectively. The The term $d^{e} S$ is the entropy supplied to the system by surrounding elements through transfer of mass and heat (e.g., in an open system where wear and corrosion mechanisms occur), which may be positive or negative. The term $d^{d} S$ is the entropy produced inside of the system, which, according to the second law of thermodynamics, must be zero for reversible transformations and positive $\left(d^{d} S \geq 0\right)$ for irreversible transformations of the system with increase of disorder.

In order to calculate the entropy generation in terms of experimentally measurable quantities, Equation (2) can be written in a local form that is appropriate for description of systems where the densities of extensive properties such as mass and energy are continuous functions of space 
coordinates $[11,46]$. As such, $S$ can be defined for a domain $g$ by the means of specific entropy $s$ per unit mass (described by density $\rho$ and volume $V$ ) as $S=\int_{g} \rho s d V$, and the rate of exchanged entropy can can be obtained as:

$$
\frac{d^{e} S}{d t}=-\int^{\Omega} \boldsymbol{J}_{s} \cdot \boldsymbol{n}_{s} d A
$$

where $\boldsymbol{J}_{S}$ is a vector of the total entropy flow per unit area per unit time (flux), crossing the boundary between the system and its surroundings, and $\boldsymbol{n}_{s}$ is a normal vector. Moreover, the rate of $d^{d} S$ can be obtained from:

$$
\frac{d^{d} S}{d t}=\int^{V} \sigma d V
$$

where $\sigma$ is the entropy generation per unit volume per unit time. Using Equations (2)-(4), and Gauss's theorem [1,11], the entropy balance, Equation (5), can be expressed in local form as:

$$
\rho \frac{d s}{d t}+\nabla \cdot \boldsymbol{J}_{s}=\sigma
$$

According to the second law of thermodynamics, the entropy generation per unit volume per unit time within the system, $\sigma$, must be zero for reversible transformations and positive for irreversible transformations of the system with increase of disorder. This suggests the use of entropy as a measure of disorder or damage ("the larger the disorder, the larger the entropy" [47]) that characterizes expenditure of the system's life. Accordingly, the damage rate of the system is closely connected to entropy generation, which accumulates progressively over time and degrades the system until its final breakdown $[2,16]$.

In order to assess damage induced in the system, every fundamental dissipative process active in the system should be identified. Using the conservation (balance laws) of energy, mass, and momentum, along with the Gibbs relation [44] and under the hypothesis of local equilibrium, the balance equation for entropy can be derived in terms of explicit expressions, as in Equation (6) $[11,48]$. The concept of local equilibrium simply assumes that for a thermodynamic system, intensive variables such as internal energy, $u$, molar number per unit volume, $n_{k}$, and specific volume, $\mathrm{v}$, that define entropy per unit mass $s\left(u, v, n_{k}\right)$ and necessarily define the macroscopic state of the system can also be well defined locally [11]. According to the local equilibrium hypothesis, it is assumed that although the total system is not in equilibrium, over a small region, there exists a state of local equilibrium where the local entropy, $s$, is the same function, $\left(s=s\left(u, \mathrm{v}, n_{k}\right)\right)$, of $u, \mathrm{v}$, and $n_{k}$, and specifically, Equation (1) remains valid.

$$
\begin{gathered}
\frac{d \gamma}{d t}=-\nabla \cdot\left(\frac{\boldsymbol{J}_{q}-\sum_{k=1}^{n}\left(c_{m} \psi+\mu_{k}\right) \boldsymbol{J}_{k}}{T}\right) \\
+\frac{1}{T^{2}} \boldsymbol{J}_{q} \cdot \nabla T-\sum_{k=1}^{n} \boldsymbol{J}_{\boldsymbol{k}}\left(\nabla \frac{\mu_{k}}{T}\right)+\frac{1}{T} \boldsymbol{\tau}: \dot{\boldsymbol{\epsilon}_{p}}+\frac{1}{T} \sum_{j=1}^{r} v_{j} A_{j}+\frac{1}{T} \sum_{m=1}^{h} c_{m} \boldsymbol{J}_{m}(-\nabla \psi),
\end{gathered}
$$

where $\gamma=\rho s$ is volumetric entropy, $\boldsymbol{J}_{q}$ is heat flux, $\boldsymbol{J}_{k}$ is diffusion flow, $\boldsymbol{J}_{m}$ represents fluxes resulting resulting from external fields (magnetic and electrical) such as electrical current, $A_{j}=-\sum_{i=1}^{u} \mu_{i} v_{j i}$ is chemical affinity or chemical reaction potential difference, $\boldsymbol{\tau}$ is the stress tensor, $\dot{\boldsymbol{\epsilon}_{p}}$ is plastic strain rate rate tensor, $\psi$ is potential of the external field such as electrical potential difference, and $c_{m}$ is the coupling constant. External forces may result from different factors including electrical fields, magnetic fields, and gravity fields where the corresponding fluxes are electrical currents, magnetic currents, and 
velocity. For example, in the case of an electric field, $E=-\nabla \psi$ is the electric potential, $\boldsymbol{I}=\Sigma_{m=1}^{h} c_{m} \boldsymbol{J}_{m}$, is current density, and $c_{m}=F z_{m}$, where $F$ is the Faraday constant and $z_{m}$ is the number of moles of electrons. Each term in Equation 6 is derived from the various mechanisms involved, which define the macroscopic state of the complete system. By comparing Equation (6) with Equation (5), we can state:

$$
\begin{gathered}
\boldsymbol{J}_{s}=\frac{\boldsymbol{J}_{q}-\sum_{k=1}^{n}\left(c_{m} \psi+\mu_{k}\right) \boldsymbol{J}_{k}}{T} \\
\sigma=\frac{1}{T^{2}} \boldsymbol{J}_{q} \cdot \nabla T-\sum_{k=1}^{n} \boldsymbol{J}_{k}\left(\nabla \frac{\mu_{k}}{T}\right)+\frac{1}{T} \boldsymbol{\tau}: \dot{\boldsymbol{\epsilon}_{p}}+\frac{1}{T} \sum_{j=1}^{r} v_{j} A_{j}+\frac{1}{T} \sum_{m=1}^{h} c_{m} \boldsymbol{J}_{m}(-\nabla \psi),
\end{gathered}
$$

where Equation (7) shows the entropy flux resulting from heat exchange and material exchange, and the presence of an external field. Equation (8) represents the total energy dissipation terms from the system that (from left to right) include heat conduction energy, diffusion energy, mechanical energy, chemical energy, and external field energy (such as magnetic, electrical, radiation, and gravity). Equation (8) is fundamental to non-equilibrium thermodynamics, and represents the entropy generation $\sigma$ as the bilinear bilinear form of thermodynamic forces and fluxes as:

$$
\sigma=\sum_{i=1}^{m} X_{i} J_{i}
$$

It is through this form that the contributions from the applicable thermodynamic forces and fluxes are expressed. To better understand the thermodynamics of materials damage, let us briefly elaborate on some fundamental thermodynamics concepts. First, we must notice that all degradation mechanisms leading to damage are in non-equilibrium states [1]. Therefore, equilibrium thermodynamics - which is merely concerned with equilibrium in which a system attains maximum entropy with zero entropy generation rate, $\sigma=0$ - cannot describe the degradation. The Classic Theory of Irreversible Thermodynamics (CTIT), on the other hand, enables us to cope with non-equilibrium changes from place to place and over time. The most important hypothesis underlying CTIT is undoubtedly the local equilibrium hypothesis. According to Lebon et al. [1], the local equilibrium hypothesis states that the local and instantaneous relations between a system's properties are the same as for a uniform system in equilibrium. Lebon et al. also suggest that systems can be divided into cells that are sufficiently large for microscopic fluctuations to be negligible but sufficiently small so that equilibrium can be realized in each individual cell to a good approximation. Based on the local equilibrium hypothesis, each cell is assumed to be in equilibrium state. Thermodynamic properties are uniform within each individual cell, but can differ among neighboring cells. Also, in each individual cell the equilibrium state can change with time [1]. Furthermore, two timescales are suggested to check the applicability of the local equilibrium assumption. The first timescale, $t_{m}$, is the time elapsed for an individual cell to attain equilibrium, and it is of the order of the time interval between two successive collisions between particles, i.e., $10^{-12} \mathrm{~s}$, at normal pressure and temperature. The second timescale, $t_{M}$, is related to the time required for macroscopic change of the system. This may be the time required for macroscopic damage induced in the system, or may be related to the duration of an experiment. The ratio of these two timescales is called the Deborah number (i.e., $D e=t_{m} / t_{M}$ ). For $D e \ll 1$, the local equilibrium hypothesis is applicable where relevant variables evolve on a large timescale $t_{M}$ and practically do not change over the time $t_{m}$. However, the hypothesis is not appropriate for describing situations for which large values of $D e$ are typical for fast or high frequency phenomena such as ultrasound propagation, shock waves, and nuclear collisions, or when other variables, not found at equilibrium, are able to influence the process. This is, for instance, 
the case for polymers found in long molecular chains where their configuration affects their activities substantially. Other examples include super-fluids and superconductors, whose irregular properties require introduction of extra variables [1]. The corrosion fatigue degradation process used as an example in this paper is considered a relatively slow phenomenon occurring over a long period and therefore justifying use of the local equilibrium hypothesis.

Qualification of the local equilibrium hypothesis for slow degradation mechanisms enabling use of Equation (5), and subsequently Equation (6), allows us to express the evolutionary trend of the total volumetric generated entropy, $\gamma_{d}$, representing damage, $D$, as:

$$
D \sim \gamma_{d} \mid t=\int_{0}^{t}\left[\sigma \mid X_{i}(u), J_{i}(u)\right] d u,
$$

where $\gamma_{d} \mid t$ is the monotonically increasing cumulative entropy starting at time zero from a theoretical value of zero or practically some initial entropy, $\gamma_{d_{0}}$, to an entropic endurance value, $\gamma_{d_{E}}$, which makes the component functionally nonperforming. When $\gamma_{d}$ reaches this entropic endurance, it may be assumed assumed that, for all practical purposes, the component or structure is considered as failed. It is worth noting that failure in this context is defined as surpassing a minimum operating requirement level for an item (the item may still be operational, but not satisfactorily). Entropic endurance is equal for the same items, but could be different for other items (structures, components, and systems). Furthermore, this entropic endurance is measurable (from experiments or field observations) and involves stochastic uncertainties due to variations in material, environmental, and operating conditions. To measure the damage in terms of the total entropy generation and the theory of damage described in this paper, a dimensionless damage index may be defined as shown in Equation (11):

$$
D=\frac{\gamma_{d}-\gamma_{d_{0}}}{\gamma_{d_{E}}-\gamma_{d_{0}}}
$$

Other examples of definitions of damage tied to entropy are introduced by Basaran et al. [23,49-52], Yan [53], Tucker et al. [54], and Chan et al. [55]. They utilized the concept of entropy from information theory [56] and statistical mechanics [57] for modeling damage and fatigue fracture initiation and growth in solder joints.

\section{Entropic-Based Damage in Corrosion Fatigue}

Corrosion fatigue is a synergetic damage mechanism where two of the relatively independent processes of corrosion and fatigue interact and accelerate the rate of damage otherwise realized by the sum of the two mechanisms. Figure 2 schematically shows the processes involved in a component subjected to the corrosion fatigue degradation mechanism, being electrochemically monitored. The corrosion process occurs at the surface of the specimen under a cyclic load with the following oxidation and reduction reactions for a metallic electrode, $M$ :

$$
\begin{gathered}
M \leftrightarrow M^{z_{M}^{+}}+z_{M} e^{-} \\
O+z_{O} e^{-} \leftrightarrow R,
\end{gathered}
$$

where $O$ is a certain oxidant in solution, which results in the formation of reduction product $R$. 


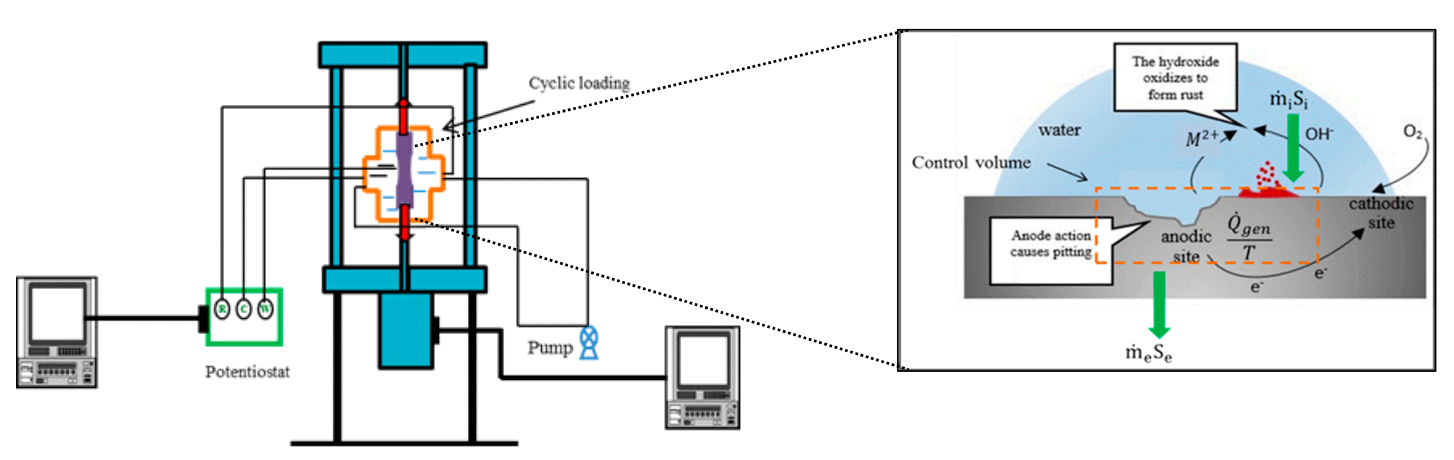

Figure 2. Entropy flow in the control volume under corrosion fatigue.

Assuming a specific control volume, the main dissipative processes during the corrosion fatigue failure mechanism include electrochemical losses induced by electronic current leakage (e.g., activation and Ohmic overpotentials); chemical reaction losses; mechanical losses; heat losses; and diffusion losses. According to Naderi et al. [21] and Ontiveros [24], the entropy generation due to heat conduction inside the solid is negligible for high-cycle fatigue. Assuming the corrosion fatigue in high-cycle regime and an adiabatic condition, the contribution of the corrosion activation overpotential, $\sigma_{\text {act }}$, corrosion reaction chemical potential, $\frac{1}{T} \sum_{j=1}^{r} v_{j} A_{j}$, diffusion overpotential, $\sigma_{c o n c}$, Ohmic losses, $\sigma_{\Omega}$, and mechanical losses, $\sigma_{M}$, on the rate of entropy generation for a control volume, can be determined by:

$$
\sigma=\sigma_{a c t}+\frac{1}{T} \sum_{j=1}^{r} v_{j} A_{j}+\sigma_{\text {conc }}+\sigma_{\Omega}+\sigma_{M},
$$

where the contribution of each term is explained in the following.

\subsection{Activation Overpotential}

Activation overpotential is the additional electric potential necessary to overcome the energy barrier in order for the electrode reaction to proceed at a desired rate. When cathodic overpotential, $E$, is applied to the electrode, the oxidation cathodic reaction rate is reduced and the oxidation anodic rate increases. This is accomplished by decreasing the activation energy for the cathodic reaction by an amount, $E_{M_{a c t, c}}=\alpha_{M} Z_{M} F\left(E-E_{c o r r}\right)$, and increasing that for anodic reaction by an amount, $E_{M_{a c t, a}}=$ $\left(1-\alpha_{M}\right) z_{M} F\left(E-E_{c o r r}\right)$, where $E_{\text {corr }}$ is the Open-Circuit Potential (OCP), and $\alpha_{M}$ is the chargetransport coefficient for oxidation reaction. Considering the thermodynamic fluxes of the anodic and cathodic half reactions in process 13 , the contribution of the corrosion activation overpotential to the entropy generation for a control volume, $V$, can be expressed as:

$$
\sigma_{a c t}=\frac{1}{T}\left(J_{M, a} z_{M} F E_{M_{a c t, a}}+J_{M, c} z_{M} F E_{M_{a c t, c}}+J_{O, a} z_{O} F E_{O_{a c t, a}}+J_{O, c} z_{O} F E_{O_{a c t, c}}\right),
$$

where $\boldsymbol{J}_{M, a}$ and $\boldsymbol{J}_{M, c}$ are the irreversible anodic and cathodic activation currents for oxidation reaction, respectively, and $\boldsymbol{J}_{O, a}$ and $\boldsymbol{J}_{O, c}$ are the anodic and cathodic activation currents for reduction reaction, respectively. In the case of corrosion fatigue, both anodic and cathodic polarization can influence the degradation mechanism, with different behaviors observed during crack initiation compared to crack growth, and for steel compared to either aluminum or titanium alloys [58]. For example, Duquette et al. [6] reported that the fatigue lives of smooth specimens of $\mathrm{Al}-7075$ and $\mathrm{Al}-\mathrm{Mg}-\mathrm{Li}$ in $\mathrm{NaCl}$ solution were reasonably low at both anodic and cathodic polarization. However, corrosion fatigue of polished 
specimens of 1020 and 4140 steels exposed to $\mathrm{NaCl}$ during high-frequency rotating bending occurred only when a critical anodic current was surpassed [58].

\subsection{Ohmic Overpotential}

The current that passes between the working electrode and auxiliary electrode creates a potential gradient in the electrolyte. This effect is called Ohmic overpotential and is more pronounced at high current densities or in electrolyte solutions of low conductivity. To minimize the effect of Ohmic overpotential, the accepted method is placement of the Luggin capillary close to the working electrode.

\subsection{Diffusion Losses}

Concentration overpotential caused by the failure of the surrounding material to preserve the initial concentration of the bulk solution as a reactant is consumed at the electrode. Concentration overpotential for anodic oxidation during corrosion can usually be ignored because of the presence of an unlimited supply of metal atoms at the interface [14]. This loss process occurs over the entire range of current density but is predominant at high cathodic reactions, leading to depletion of the dissolved species in the adjacent solution [59]. The contribution of the concentration overpotentials for cathode reaction, using a simplified approach based on Fick's law, can be obtained as:

$$
\sigma_{\text {conc }}=\frac{1}{T}\left(\boldsymbol{J}_{M, c} E_{M_{\text {conc }, c}}+\boldsymbol{J}_{O, c} E_{O_{c o n c, c}}\right) \text {, }
$$

where $E_{M_{c o n c, c}}$ and $E_{O_{c o n c, c}}$ are cathodic oxidation and reduction diffusion overpotentials, respectively.

\subsection{Mechanical Losses}

The mechanical dissipative losses, $\sigma_{M}$, constitute plastic, $\dot{\boldsymbol{\epsilon}_{\boldsymbol{p}}}: \boldsymbol{\tau}$, and elastic energy losses. Plastic losses are explicitly associated with the total generated entropy as shown in Equation (8). When the material is under cyclic loading in the elastic region, consistent with the continuum mechanic rules, it does not experience any permanent deformation, and the stress-strain diagram goes back to the origin [52]. In other words, fatigue would not be possible under the elastic regime, which would violate the second law of thermodynamics. Inspection of the material at the atomic scale, however, shows that atoms do not necessarily return to their initial lattice site but to a lower energy level [60], which leads to the formation of nanoscale voids and stress concentration locations. As a result, these voids grow and coalesce to form micro-cracks in the material. For the case of high-cycle fatigue under elastic deformation, Ontiverous [24] and Temfack and Basaran [52] introduce irreversible entropy generation due to elastic work. Due to the mechanical nature of the fracture and crack propagation, we categorize losses due to these degradation processes as resulting from mechanical losses.

Interactions between dislocations and the environment at the crack initiation points are commonly suggested as a source of degradation in corrosion fatigue [58]. The hydrogen embrittlement hypothesis is supported by extensive but incidental evidence as an important mechanism for corrosion fatigue crack propagation, and the hydrogen embrittlement hypothesis is the most readily acknowledged degradation mechanism for high-strength alloys in strong hydrogen-producing environments [61-63]. Hydrogen embrittlement results from the diffusion of hydrogen atoms (a byproduct of the corrosion process) into 
the lattice space of metals and alloys, which can weaken atomic bonds, causing de-cohesion of lattice bonds and enhancing localized plasticity through crack tip dissolution. Hydrogen-enhanced corrosion fatigue cracking is either inter-granular or trans-granular, with the latter involving a dislocation of the substructure [58].

A thermodynamic model for hydrogen-enhanced anodic dissolution was first suggested by Qiao et al. [64]. Near-neutral stress corrosion cracking analysis in some studies [65,66] has suggested the acceleration of anodic current due to hydrogen-facilitated free energy increments and strong synergistic interactions between the dissolved hydrogen and the local stress field around the crack tip. However, such a strong change in the free energy and synergistic effect is not supported by the experimental results found by other studies [67-69].

Another important mechanism for initiating cracks is when passive films are not capable of extensive plastic deformation relative to the underlying metal, which can cause corrosion fatigue damage through one or more processes, including a reduction of near-surface plasticity, leading to reduced or enhanced corrosion fatigue (depending on the cracking mechanism), localization of near-surface dislocation structure and voids, and film-induced cleavage [58,70,71]. These phenomena-along with others such as micro-crack propagation [72-74], surface energy change [75], crack closure [76,77], microstructure [78], crack size [79], and configuration of micro-cracks and cavities - represent internal corrosion fatigue processes requiring an advanced means of measurement by direct observation, and do not appear explicitly either in the law of conservation of energy or the second law of thermodynamics. The internal variables theory [1], an alternative approach to non-equilibrium thermodynamics, enables us to supplement local equilibrium variables (e.g., volume and deformation) with the corresponding scalar or tensorial variables that represent these internal phenomena. It is beyond the scope of this study to analyze these phenomena by implementing internal variables, but benefitting from the entropy characteristic that "as a measure of uncertainty" [22] comprises the variabilities in the microstructural states [54], their subsequent effects are considered in the stress-strain and corrosion potential-electrical charge hysteresis energy loops described in the next section.

\subsection{Total Entropy Generation during Corrosion Fatigue}

Using Equation (14), the contribution of the above factors to the entropy generated in a control volume can be expressed as:

$$
\begin{gathered}
\sigma=\frac{1}{T}\left(\boldsymbol{J}_{M, a} \alpha_{M} \tilde{A}_{M}+\boldsymbol{J}_{M, c}\left(1-\alpha_{M}\right) \tilde{A}_{M}+\boldsymbol{J}_{O, a} \alpha_{O} \tilde{A}_{O}+\boldsymbol{J}_{O, c}\left(1-\alpha_{O}\right) \tilde{A}_{O}\right)+ \\
\frac{1}{T}\left(\boldsymbol{J}_{M, c} E_{M_{c o n c, c}}+\boldsymbol{J}_{O, c} E_{O_{c o n c, c}}\right)+\sigma_{\Omega}+\frac{\boldsymbol{\epsilon}_{\boldsymbol{p}}: \boldsymbol{\tau}}{T}+\frac{\sigma_{M e}}{T},
\end{gathered}
$$

where $\tilde{A}=\Sigma_{i} v_{i} \tilde{\mu}_{i}$ is electrochemical affinity induced by electrochemical potential $\tilde{\mu}_{i}=\mu_{i}+$ $z_{i} F\left(E-E_{c o r r}\right)$, and $\sigma_{M_{e}}$ is the dissipative part of the energy resulting from elastic deformation of material.

\section{Materials and Experimental Procedures}

The material being investigated is the cold-rolled alloy aluminum 7075-T6. The specimen was designed using finite element analysis to ensure that failure occurred in the middle section with a width of $2 \mathrm{~mm}$. The structural mechanics module of COMSOL software was used to perform a finite element 
analysis in which the specimen used in this research was analyzed under static tension load. In this simulation, the linear elastic model is used to describe the material. Figure 3 shows the design and von Mises stress distribution of the Al 7075-T6 specimen under an axial tensile load with the maximum values in the center of the specimen.

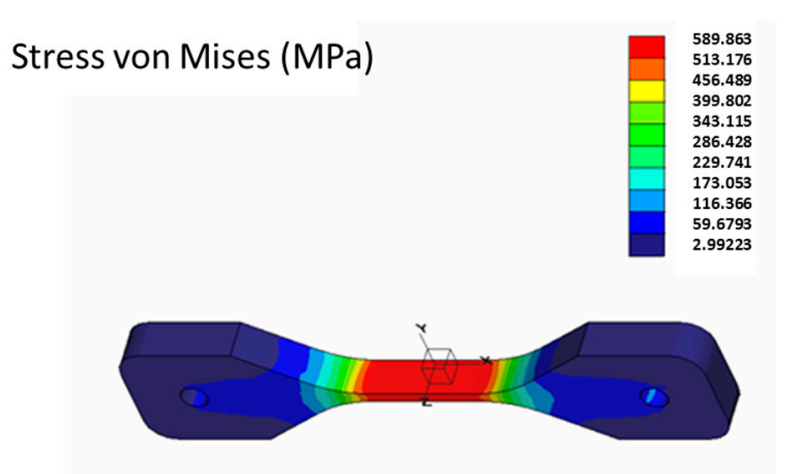

(a)

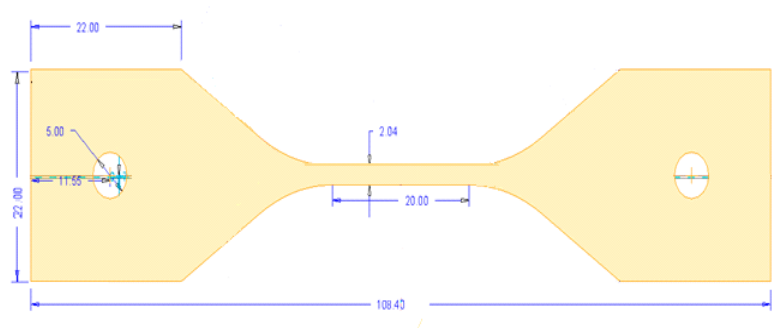

(b)

Figure 3. (a) Al 7075-T6 finite element analysis; (b) specimen cross section with thickness of 6.3 (dimensions are in $\mathrm{mm}$ ).

Fatigue tests were carried out in a corrosive $3.5 \mathrm{wt} \% \mathrm{NaCl}$ aqueous solution, acidified with a 1 molar solution of $\mathrm{HCl}$, with a $\mathrm{pH}$ of 3 , under axial load/unload controlled under free corrosion potential. Before testing, the specimens were ground with $\mathrm{SiC}$ abrasive papers of grits up to \#1200, then successively cleaned and rinsed with de-ionized water and acetone. The schematic of the experimental setup is shown in Figure 4a. The Instron software recorded mechanical stress and strain values. A digital image correlation technique [80] was also employed to validate Instron outputs. An electrochemical corrosion cell, made from plexiglass, was designed and built to be compatible with the whole gauge section of the specimen as shown in Figure 4b. The whole gauge section (the area of interest) of the specimen was electrochemically monitored using a Gamry potentiostat device with an $\mathrm{Ag} / \mathrm{AgCl}$ electrode as the reference electrode $(\mathrm{RE})$ maintained at a constant distance $(2 \mathrm{~mm})$ from the specimen, a platinum counter electrode (CE) and the specimen as the working electrode (WE). All the tests were conducted after the WE, immersed in the solution, reached the equilibrium OCP.

Before the principle corrosion fatigue tests were conducted, a tensile mechanical test was performed to obtain the mechanical and electrochemical properties of the specimen immersed in corrosive environment. Figure 5 shows the decrease of elongation to fracture of a specimen tested in the $\mathrm{NaCl}$ $3.5 \mathrm{wt} \%$ solution versus air. Alexopoulos et al. [81] show the same property for a tensile test of Aluminum 2024 in a corrosive environment. The yield and ultimate strength of the corroded specimen decreased by $20 \%$ when the tensile strength of the specimen was tested in air.

The corrosion fatigue tests were conducted while measuring the OCP variations during load/unload versus the unstrained RE. Figure 6 shows a dependence of OCP of a specimen under cyclic load as a result of mechano-chemical effect. According to the mechano-chemical effect, we can expect to observe an enhanced anodic dissolution flux induced by dynamic surface deformation. Meanwhile, the anodic current at the electrode surface can decrease near-surface work hardening and increase the mobility of dislocation, ultimately stimulating fatigue damage $[14,15,58]$. The strain response of electrode potential or work function has long been of interest in relation to a wide range of problems, including corrosion 
in structural materials under load [14,82-84] and coupling of electronic properties to stress in semiconductor devices [85,86], and to diffusion [87,88] and metallurgy [89]. Gutman [14] and Sahal et al. [84] suggested that the local value of the chemical potential in the liquid/solid system is defined only by the absolute value of the hydrostatic part of the stress tensor, $\tau_{m}$, and is independent of the deviatory part of the stress. Using the solid incompressibility hypothesis [14], the potential difference caused by stress is given by:

$$
\Delta \phi=\frac{\Delta V \tau_{m}}{z_{M} F}=V_{m} \tau_{m}
$$

where $V_{m}$ is the molar volume. It should be noted that the increase of the free energy due to the field stress only influences the potential of the ions in the metal, and not the potential of the metal ions in the electrolyte. If the Ohmic and concentration overpotentials are considered negligible, the dissolution current density can be expressed as [14]:

$$
i=J_{M}^{0} \exp \left(\frac{V_{m} \tau_{m}}{R T}\right) \exp \left(\frac{\alpha_{M} \tilde{A}_{M}}{R T}\right)-J_{M}^{0} \exp \left(\frac{\left(1-\alpha_{M}\right) \tilde{A}_{M}}{R T}\right),
$$

where $\exp \left(\frac{V_{m} \tau_{m}}{R T}\right)$ expresses the effect of stress. In this paper, the value of $\Delta \phi$ is obtained in a useful range, in accordance with the experimental results. For example, in Figure 6, where the maximum cyclic stress, $P=330 \mathrm{MPa}$, is applied, the potential difference, $\Delta \phi=0.002 \mathrm{v}$, is in good agreement with the value of $\Delta \phi$ obtained from Equation 18 , where molar volume, $V_{m}=10 \mathrm{~cm}^{3} / \mathrm{mol}$, the hydrostatic part of of the stress tensor, $\tau_{m}=\frac{1}{3} P$ [14], and $z_{M}=3$ were considered for the anodic dissolution of the aluminum matrix $[59,90]$, which is believed to dissolve when it is immersed in $3.5 \mathrm{wt} \% \mathrm{NaCl}$ solution. For Aluminum 7075, whose main constituent particles include C-type, A-type, and silicon-containing particles, it has been shown [91,92] that when it is immersed in $3.5 \% \mathrm{NaCl}$ solution, there is no substantial attack on the matrix that surrounds C-type particles; however, at the A-type particle sites, apparent attack was observed. Because of the more negative corrosion potential of these particles relative to the matrix, they start to dissolve. However, due to the selective dissolution of $\mathrm{Mg}$ in A-type particles $\left(\mathrm{Al}_{2} \mathrm{CuMg}\right)$, the remaining particles may become $\mathrm{Cu}$-rich and cathodic relative to the matrix. In these cases, the surrounding aluminum matrix will be under corrosion pitting attack [91].

Tests were carried out at the maximum cyclic stress, $P$, levels of $87 \%, 80 \%$, and $70 \%, 63 \%$ and $57 \%$ of the corroded specimen yield stress; stress ratio of 0.01 ; and loading frequency of $0.04 \mathrm{~Hz}$. The stop criterion was the failure of specimens.

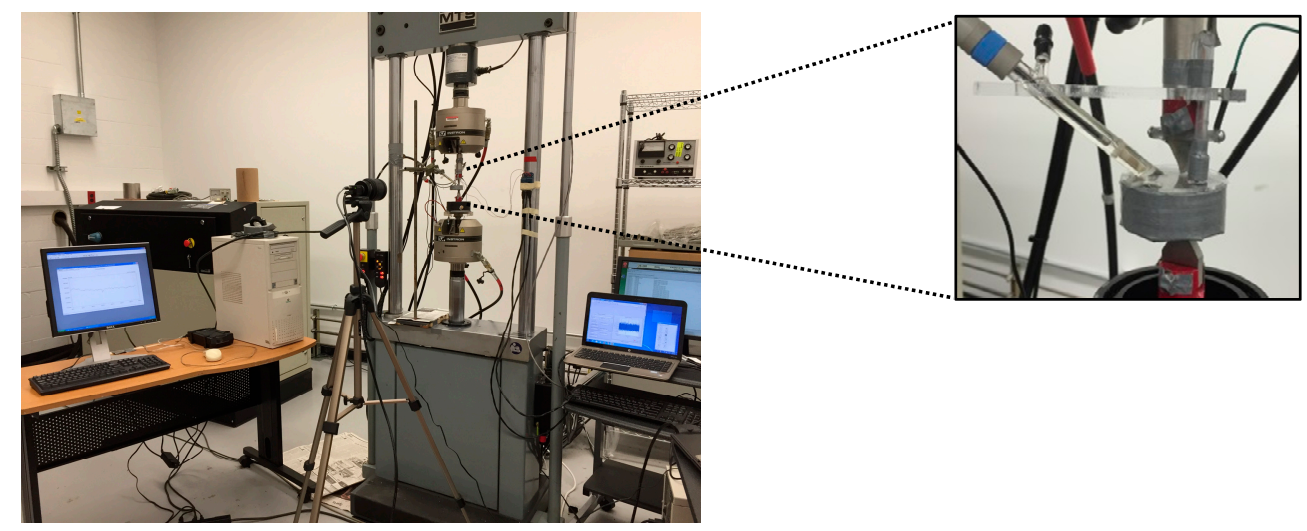

(a)

(b)

Figure 4. Experimental setup. 


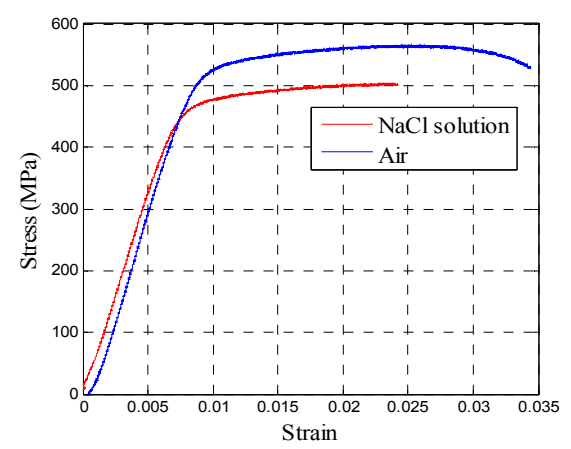

Figure 5. Tensile stress-strain curves for aluminum alloy 7075-T651 in air and $\mathrm{NaCl} 3.5 \mathrm{wt} \%$.

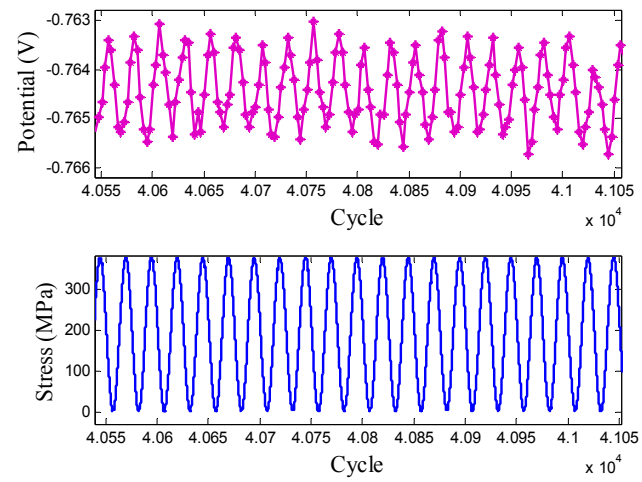

Figure 6. Open circuit potential variation during cyclic load application.

\section{Quantification of Entropy in Corrosion Fatigue}

In this study, the corrosion fatigue entropy generation was quantified based on the following assumptions: (1) the corrosion process occurs close to the equilibrium state (i.e., OCP), and the classical theory of irreversible processes does not cope with situations far from equilibrium; (2) entropy flow due to heat exchange is negligible in accordance with Naderi et al. [21] and Ontiveros [24], whose results showed insignificant entropy generation due to heat conduction inside the solid under high-cycle fatigue. Although the high-cycle fatigue does not result in significant heat conduction, low-cycle fatigue can experience noticeable thermal heat generation and conduction [93]; (3) diffusion losses can be eliminated since the solution species are assumed to be well mixed; (4) the effects of diffusivity and concentration of hydrogen at the crack surface can be excluded from entropy calculation because, for aluminum alloys under cyclic loading in the sodium chloride solution, Mason [94] indicates that above a critical frequency of load cycling (of $0.001 \mathrm{~Hz}$ ), shorter cycle periods provide less time for diffusion and accumulation of hydrogen; (5) the Ohmic overpotential can be ignored, as its effect was minimized by placement of the Luggin capillary close to the working electrode; and (6) the corresponding current to OCP records can be obtained based on the fit of the sum of two exponential functions to the part of the polarization curve where OCPs vary (i.e., around the second breakdown potential) in the corrosion fatigue experiments (refer to Figure 7a,b). In order to explore the effect of corrosion time and cyclic loading on the polarization behavior, a potentiodynamic scan was performed at different loads and immersion time periods, as represented in Figure 7a,c. Since the results show no particular patterns in the variation of 
polarization curves with alteration of load conditions and immersion times, the mean of the polarization curves with a greater range of uncertainty (Figure 7a) was used for corrosion current evaluation.

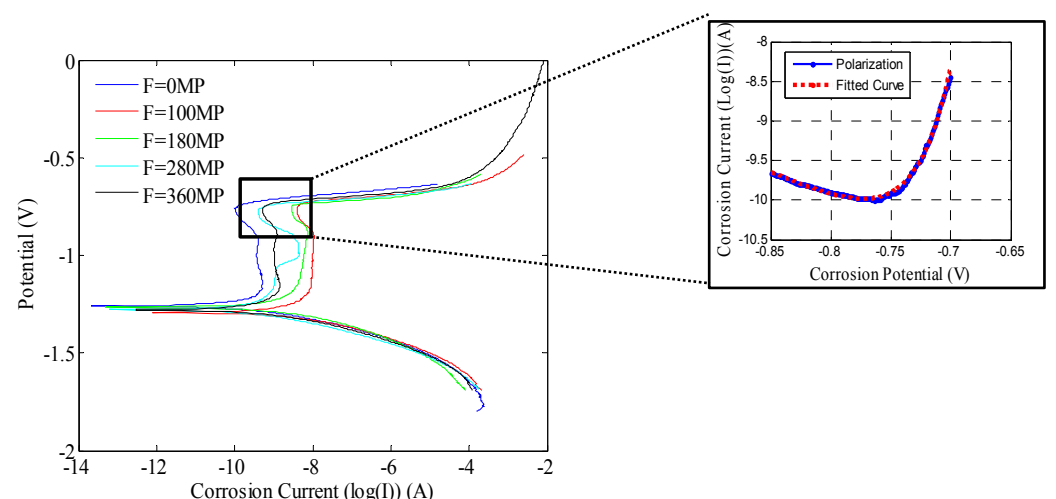

(a)

(b)

Figure 7. (a) Polarization curves at different stress levels; (b) the corrosion current as a function of potential was obtained by fitting the sum of two exponential functions to the interval of potential values corresponding to OCP variations during corrosion fatigue tests; (c) polarization curves after different immersion time periods.

The above assumptions allow us to eliminate the effects of heat conduction, diffusion, hydrogen embrittlement, and Ohmic overpotential due to their insignificant contributions to entropy generation. The amount of entropy generated in each cycle of the corrosion fatigue experiment is then obtained in terms of the area of the hysteresis energy loops resulting from mechanical stress-strain [95] and the energy resulting from variation of corrosion potential-current in each cycle of loading and unloading. Results are shown in Figure 8.
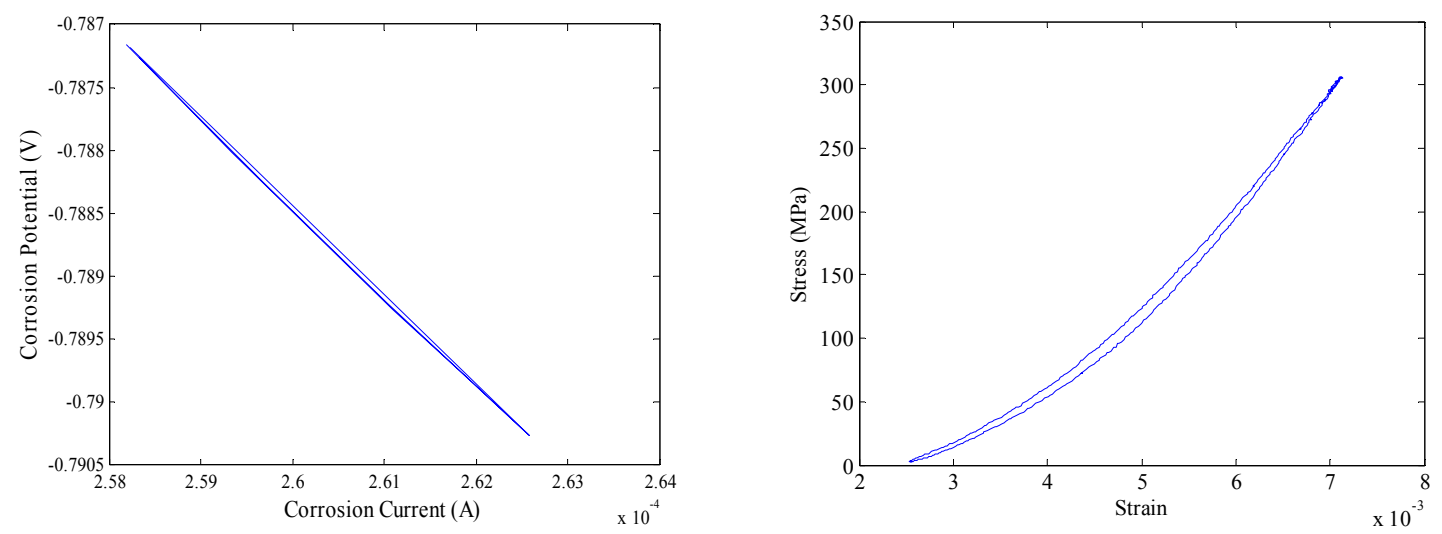

Figure 8. Stress-strain and corrosion potential-current hysteresis energy loops.

Figure 9a presents the evolution trend of corrosion fatigue volumetric entropy for different loading conditions. Filled markers show the final value of fracture corrosion fatigue entropy. The results show that the cumulative entropy values at the fracture points remain between $0.7 \mathrm{MJm}^{-3} \mathrm{~K}^{-1}$ and $1.5 \mathrm{MJm}^{-3} \mathrm{~K}^{-1}$. Figure $9 \mathrm{~b}$ presents a box plot of the fracture entropies at each loading condition, revealing that the mean of the fracture entropy values remains roughly $1.05 \mathrm{MJm}^{-3} \mathrm{~K}^{-1}$ with a standard deviation of 0.2 . These results justify the independence of entropy to the loading condition, although 
fracture entropies build a narrow distribution. Existence of the fracture entropy distribution can be explained by the presence of inherent uncertainties such as instrumental measurement errors, the legitimacy of the assumptions considered in entropy evaluation, weak control of the experimental, operational and environmental conditions, and human error. Figure 10 presents the normalized corrosion entropy with respect to the normalized fatigue entropy, where normalized entropies were calculated by dividing the entropy resulting from corrosion and fatigue, respectively, to the total entropy gain at the final fracture for different loading conditions. The result, interestingly, suggests that the normalized corrosion entropies increase monotonically with decreases in load, compensating for the decreases in fatigue entropy.

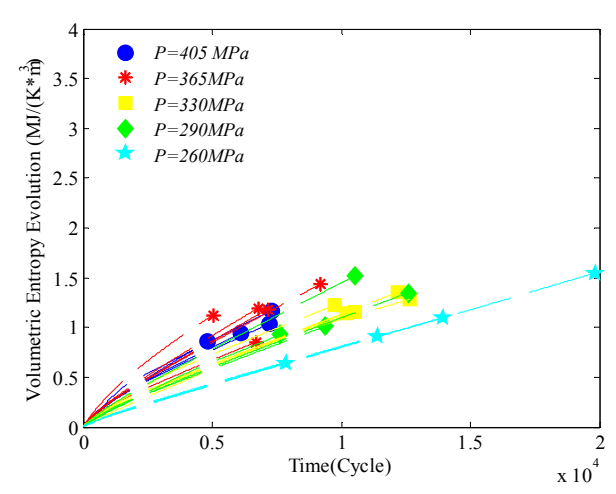

(a)

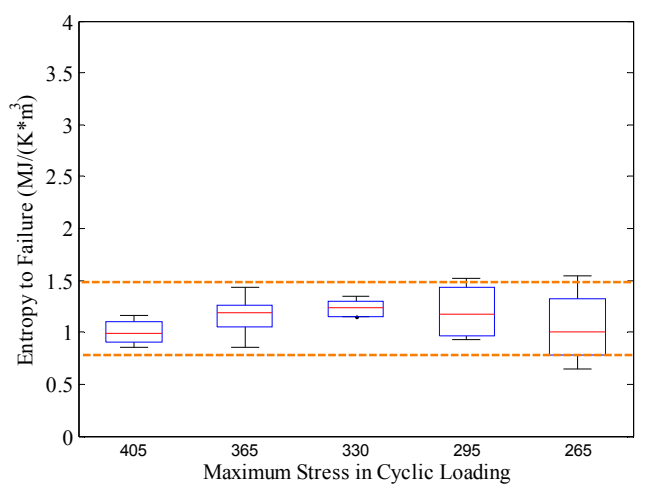

(b)

Figure 9. (a) Cumulative entropy evolutions at different loading conditions; (b) box plot of fracture entropies (entropy to failure) at each loading condition where boxes represent the interval of fracture entropy distributions, and mid lines reflect the mean of fracture entropies at each loading condition.

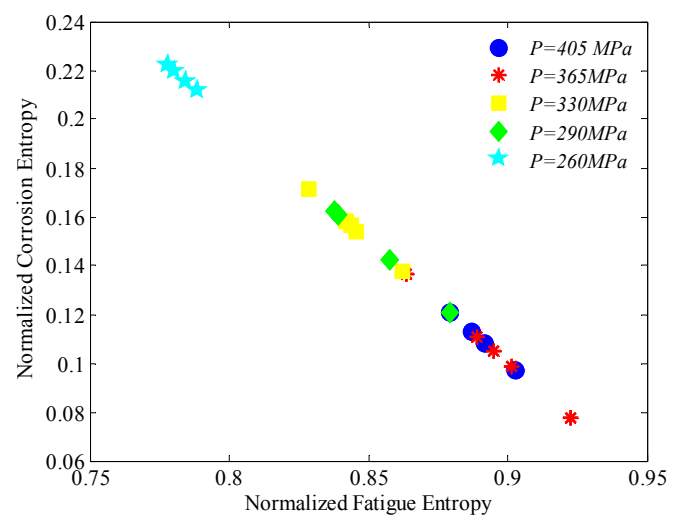

Figure 10. Normalized corrosion entropy versus normalized fatigue entropy.

\section{Entropic-Based Reliability Assessment}

As previously discussed, material, environmental, operational, and other variabilities in degradation processes impose uncertainties on the damage trends $D$ (normalized entropy), and time-to-failure (TTF) data points as depicted by Figure 11. This figure generally shows how the cumulative damage curves starting from an initial level of zero reach the damage threshold, $D_{f}$, equal to one. The variations in the resulting times can be described by a random variable and described as the probability density function 
(PDF) of the times-to-failure. This PDF can then be used to describe the life expended and the useful remaining life of the structure. Mathematically, the probability that the random variable $D$ (i.e., the cumulative damage at the observed failure time, $t_{c}$ ), exceeds $D_{f}$ (the normalized "entropicendurance" discussed earlier) is equal to the probability that the random variable $T_{r}$ (time-to-failure) is less than $t_{c}$. Accordingly, one can derive the time-to-failure PDF, $g(t)$, from the thermodynamic index of damage (i.e., cumulative entropy) described by the PDF $f(D)$ :

$$
P_{r}\left(T_{r} \leq t_{c}\right)=\int_{0}^{t_{c}} g(t) d t=1-\int_{0}^{D_{f}=1} f(D) d D,
$$

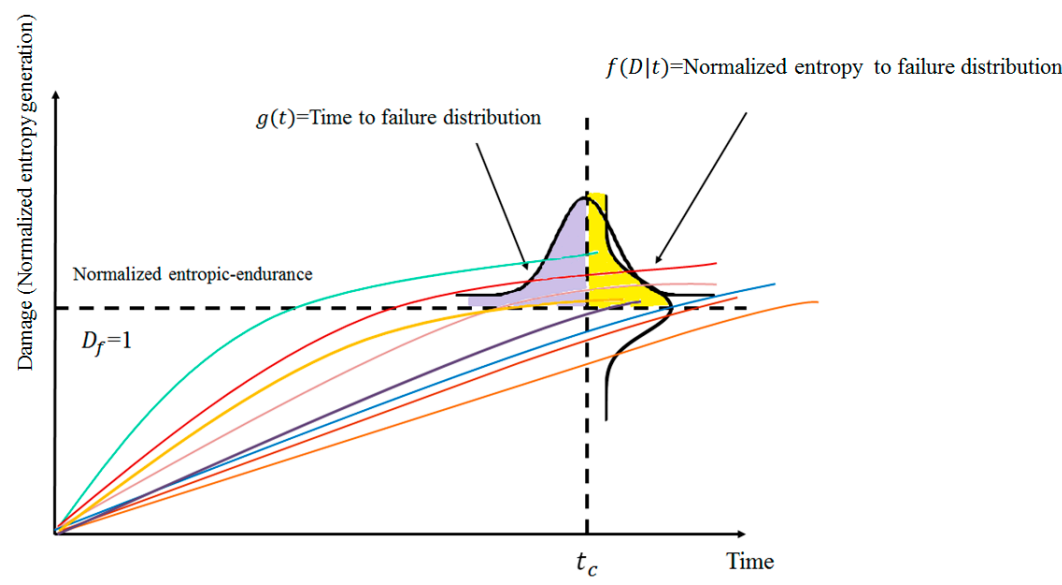

Figure 11. Distribution of damage-to-failure data points and time-to-failure data points.

Using Equation (20) and the linear relationship between the cycle's evolution and cumulative entropies resulting from corrosion fatigue experiments shown in Figure 12a, the CTF PDF, $(g(N))$, can be derived in terms of the normalized entropy-to-failures (NETF) PDF, $(f(D))$ as expressed in Equation (21). Note that in Equation (21), the number of cycles, $N$, represents time.

$$
g(N)=\frac{\lambda}{N^{2}} f\left(\frac{\lambda}{N}\right)
$$

where $\lambda=D_{f} t_{c}$ is the CTF distribution parameter. Figure $12 \mathrm{c}$ represents the derivation of CTF PDF by applying Equation 21 to the NETF PDF, shown in Figure 12b. While the empirical distribution of NETF would be sufficient, one could also seek known distribution models that fit well into the NETF data. In this case, it appears that the Weibull distribution fits reasonably well to the corrosion-fatigue NETF data points obtained in these tests at the failure time, $t_{c}$, with a coefficient of determination, $R^{2}$, equal to 0.92 . 0.92. By substitution of the Weibull distribution in Equation (21), the CTF PDF can be derived accordingly. The sum of square error of 0.0008 between the derived PDF and the true CTF PDF (refer to Figure 12c) signifies the goodness of fit. Based on the derived CTF PDF, the reliability of the specimens was estimated as $60 \%$. In these calculations, $t_{c}$ is selected as the mean of CTF (9493 cycle) data points. 


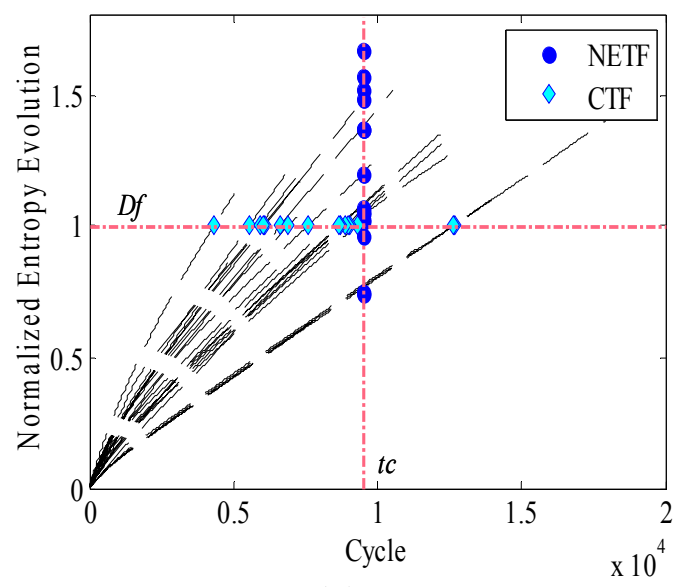

(a)

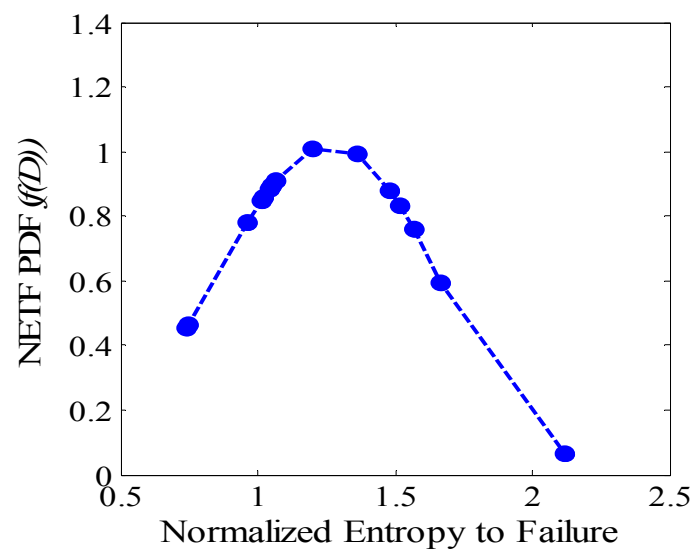

(b)

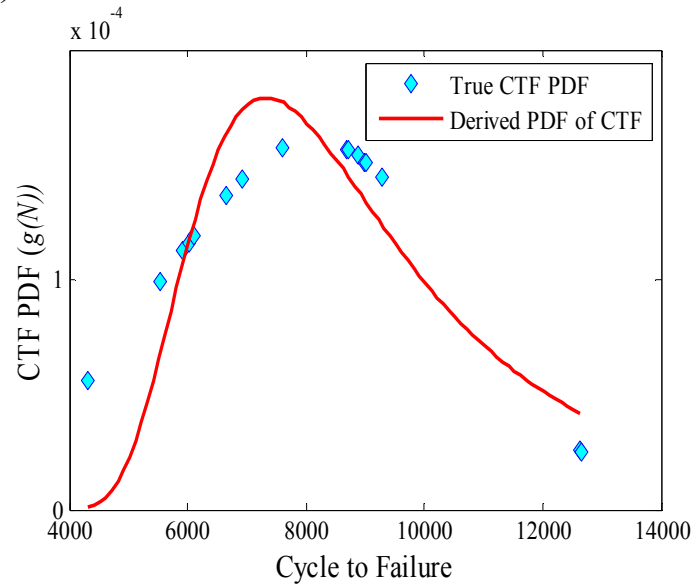

(c)

Figure 12. (a) Normalized entropy evolution of aluminum specimens; filled diamond and filled circles show the CTF and NETF; respectively. (b) Weibull distribution function was fitted to the NETF; (c) derived PDF of CTF based on NETF PDF versus true CTF distribution.

Currently, most health management processes are based on common reliability analysis and maintenance scheduling using historical data on the TTF distribution of components and structures. In this paper, however, we showed that reliability function could be derived from the total thermodynamic entropy generated rather than estimated from historical TTF data. Applying thermodynamics entropy offers a science-based approach to the definition and assessment of damage that reveals the contribution of the underlying failure mechanism, e.g., fatigue, corrosion, heat losses, etc., to the overall failure. In many cases the conventional reliability analysis is neither sufficient nor efficient because each of the components can undergo different life cycles and hence different rates of aging. Therefore, if maintenance or replacement is done solely based on reliability analysis, in most circumstances the components will either be abandoned before they have reached the end of their life or, worse, they will fail before their scheduled replacement. Prognostics and health management modeling approaches, on the other hand, provide warnings before failures happen. They also enhance the maintenance schedule, reduce the life cycle cost of inspection, and improve qualification tests to assist in design and manufacturing. Traditionally, these methods rely on the condition of the performance data that are evaluated by empirically based physics-of-failure models [94,96-98] or data-driven techniques such as 
machine learning algorithms [99-101]. The physics-of-failure-based prognostics methods employ observable makers of damage such as life cycle loading profile, material properties, and geometry for prediction purposes, but, as discussed earlier, these are only partial pictures of the entire damage. In addition, it is difficult for these methods to capture the interactions among multiple failure mechanisms. Data-driven models, on the other hand, are able to obtain the complex relationships and degradation trends in the data without having to rely on physical models, such as models of degradation or material properties. However, they cannot identify the underlying differences between different failure modes and mechanisms. Imanian and Modarres [102] have proposed an entropic-based prognostics framework to estimate the RUL of critical components and structures that not only offers a science-based foundation for prognostic methods, but also benefits from the superior advantages of the use of entropy as a damage index, in contrast with the common observable markers of degradation. Furthermore, constant entropy gain properties at failure points can effectively handle the uncertainties related to failure threshold [103,104], which has a critical effect on RUL prediction performance. As the next step, to improve the structural integrity analysis and RUL estimation, we intend to implement the so-called entropic-based prognostic framework to the corrosion fatigue degradation mechanism simulated in this study.

\section{Conclusions}

This paper discusses an approach for characterizing an index of damage on the basis of the generated entropy and describes and derives integrity, reliability, and risk expressions of critical components and structures within the irreversible thermodynamic framework. It suggests that a unified index of damage can be defined based on the entropy generation concept, which is capable of capturing the effects of multiple competing and interacting failure mechanisms. Furthermore, entropy, as a state function, is independent of the failure path, providing a favorable means for structural integrity assessment.

To evaluate the practicality of the approach for reliability analysis, the proposed approach was applied to the corrosion fatigue degradation mechanism in a laboratory environment. The thermodynamic entropies generated in the corrosion fatigue degradation mechanism of dog-bone samples were evaluated in terms of the associated dissipative processes. The experimental results showed that the cumulative entropy generations at the time of failure, while scattered due to material-to-material variability, remain constant and are independent of the loading conditions. It was shown that the contribution from the cumulative corrosion entropy increased with the decrease of load (which corresponds to the decrease of fatigue entropy), but that the total cumulative entropy remained constant. Furthermore, an alternative approach for reliability assessment based on the second law of thermodynamics was proposed, in which the reliability function was derived based on the relationship between the NETF PDF and CTF PDF.

Although the use of entropy for degradation assessment and reliability analysis, in the special case of corrosion fatigue mechanism simulated in the lab, showed satisfactory outcomes for fulfilling the aims of this paper, the advantages of this method remain to be explored for different materials, various operational conditions (e.g., different loadings, loading frequencies, loading ratios and activation over potentials), different geometries (e.g., different sizes and shapes), various environmental conditions (e.g., different temperatures, corrosive solution PHs, solution flow rates and radiations), and different failure mechanisms. This approach could open the window to further exploration of the applications of the 
thermodynamics for reliability and integrity assessment and prognosis and health management of critical components and structures.

\section{Acknowledgments}

This work is part of ongoing research through grant number N000141410005 from the Office of Naval Research (ONR).

\section{Author Contributions}

Both authors contributed equally to the development of this paper.

\section{Conflicts of Interest}

The authors declare no conflict of interest.

\section{Nomenclature}

\begin{tabular}{|c|c|c|}
\hline Parameter & Unit & Description \\
\hline$A_{i}$ & $\mathrm{~J}$ & Chemical affinity $(i=1,2, \ldots)$ \\
\hline$\tilde{A}_{M}$ & $\mathrm{v}$ & Electrochemical affinity of oxidation reaction \\
\hline$\tilde{A}_{O}$ & $\mathrm{v}$ & Electrochemical affinity of reduction reaction \\
\hline$D$ & Unitless & Damage \\
\hline$d$ & Unitless & Superscript for dissipative part of the entropy \\
\hline$D_{f}$ & Unitless & Damage threshold \\
\hline$d^{e} S$ & $\left(\mathrm{~J} \mathrm{~K}^{-1}\right)$ & Variation of exchange entropy \\
\hline$d^{d} S$ & $\left(\mathrm{~J} \mathrm{~K}^{-1}\right)$ & Variation of dissipative entropy \\
\hline E & $\mathrm{v}$ & Applied overpotential \\
\hline$E_{\text {corr }}$ & $\mathrm{v}$ & Electrode open circuit potential \\
\hline$E_{M_{a c t, a}}$ & $\mathrm{v}$ & Anodic overpotential for oxidation reaction \\
\hline$E_{M_{a c t, c}}$ & $\mathrm{v}$ & Cathodic overpotential for oxidation reaction \\
\hline$E_{O_{a c t, a}}$ & $\mathrm{v}$ & Anodic overpotential for reduction reaction \\
\hline$E_{O_{a c t, c}}$ & $\mathrm{v}$ & Cathodic overpotential for reduction reaction \\
\hline$E_{M_{\text {conc }, c}}$ & $\mathrm{v}$ & Concentration overpotential for cathodic oxidation \\
\hline$E_{O_{\text {conc }, c}}$ & $\mathrm{v}$ & Concentration overpotential for cathodic reduction \\
\hline$e$ & Unitless & Superscript for exchange part of the entropy \\
\hline$F$ & $\mathrm{C} \mathrm{mol}^{-1}$ & Faraday's constant \\
\hline$I$ & A & Electrical current \\
\hline$J_{s}$ & $\mathrm{~J} \mathrm{~K}^{-1} \mathrm{~m}^{-2} \mathrm{~s}^{-1}$ & Total entropy flow per unit area per unit time \\
\hline$J_{q}$ & $\mathrm{~W} \mathrm{~m}^{-2}$ & Heat flux \\
\hline$J_{k}$ & $\mathrm{~mol} \mathrm{~m} \mathrm{~s}^{-1}$ & Diffusion flux \\
\hline$J_{M, a}$ & A & Irreversible flux of anodic half-reaction of oxidation \\
\hline$J_{M, c}$ & A & Irreversible flux of cathodic half-reaction of oxidation \\
\hline$J_{O, a}$ & A & Irreversible flux of anodic half-reaction of reduction \\
\hline
\end{tabular}




\begin{tabular}{|c|c|c|}
\hline$J_{O, c}$ & A & Irreversible flux of cathodic half-reaction of reduction \\
\hline$N$ & Cycle & Number of fatigue load cycles \\
\hline$n_{k}$ & $\mathrm{~mol} \mathrm{~m}^{-3}$ & Molar number per unit volume \\
\hline$P$ & $\mathrm{~Pa}$ & Maximum stress in cyclic load-unload \\
\hline$P_{r}$ & Unitless & Probability \\
\hline$S$ & $\mathrm{~J} \mathrm{~K}^{-1}$ & Entropy \\
\hline$s$ & $\mathrm{~J} \mathrm{~kg}^{-1} \mathrm{~K}^{-1}$ & Specific entropy \\
\hline$T$ & $\mathrm{~K}$ & Temperature \\
\hline$T_{c}$ & Cycle & Endurance level \\
\hline$T_{r}$ & s & Time random variable \\
\hline$u$ & $\mathrm{Jm}^{-3}$ & Specific energy \\
\hline$V$ & $\mathrm{~m}^{3}$ & Volume element \\
\hline$V_{m}$ & $\mathrm{~m}^{3} \mathrm{~mol}^{-1}$ & Molar volume \\
\hline $\mathrm{v}$ & Unitless & Specific volume \\
\hline$v_{i}$ & $\mathrm{~mol} \mathrm{~s}^{-1}$ & Chemical reaction rate \\
\hline$z_{M}$ & Unitless & Number of moles of electrons exchanged in the oxidation process \\
\hline$z_{O}$ & Unitless & Number of moles of electrons exchanged in the reduction process \\
\hline$\alpha_{M}$ & Unitless & Charge transport coefficient for the oxidation process \\
\hline$\alpha_{O}$ & Unitless & Charge transport coefficient for the reduction process \\
\hline$\gamma$ & $\mathrm{J} \mathrm{m}^{-3} \mathrm{~K}^{-1}$ & Volumetric entropy \\
\hline$\gamma_{d}$ & $\mathrm{~J} \mathrm{~m}^{-3} \mathrm{~K}^{-1}$ & Volumetric entropy generation \\
\hline$\gamma_{d_{E}}$ & $\mathrm{~J} \mathrm{~m}^{-3} \mathrm{~K}^{-1}$ & Entropic-endurance \\
\hline$\epsilon_{p}$ & Unitless & Plastic strain \\
\hline$\lambda$ & Cycle & Distribution parameter \\
\hline$\mu_{k}$ & $\mathrm{~J} \mathrm{~mol}^{-1}$ & Chemical potential \\
\hline$\tilde{\mu}_{k}$ & $\mathrm{~J} \mathrm{~mol}^{-1}$ & Electrochemical potential \\
\hline$\rho$ & $\mathrm{kg} \mathrm{m}^{-3}$ & Density \\
\hline$\sigma$ & $\mathrm{J} \mathrm{m}^{-3} \mathrm{~K}^{-1}$ & Entropy generation per unit volume per unit time \\
\hline$\sigma_{\text {act }}$ & $\mathrm{J} \mathrm{m}^{-3} \mathrm{~K}^{-1}$ & Entropy generation due to activation losses per unit volume per unit time \\
\hline$\sigma_{\text {conc }}$ & $\mathrm{J} \mathrm{m}^{-3} \mathrm{~K}^{-1}$ & Entropy generation due to concentration losses per unit volume per unit time \\
\hline$\sigma_{\Omega}$ & $\mathrm{J} \mathrm{m}^{-3} \mathrm{~K}^{-1}$ & Entropy generation due to Ohmic losses per unit volume per unit time \\
\hline$\tau$ & $\mathrm{Pa}$ & Stress tensor \\
\hline$\tau_{m}$ & $\mathrm{~Pa}$ & Hydrostatic part of the stress \\
\hline$v$ & Unitless & Stoichiometric coefficient \\
\hline$\psi$ & $\mathrm{V}$ & Potential of the external field \\
\hline
\end{tabular}

\section{References}

1. Lebon, G.; Jou, D.; Casas-Vázquez, J. Understanding Non-Equilibrium; Springer: Berlin, Germany, 2008.

2. Amiri, M.; Modarres, M. An Entropy-Based Damage Characterization. Entropy 2014, 16, 6434-6463. 
3. Lemaitre, J. A Course on Damage Mechanics; Springer: Berlin, Germany, 1996.

4. Arson, C. Poromechanics of Damage and Healing: A Philosophy of the Mesoscale; Newsletter of ASCE Engineering Mechanics Institute: Reston, VA, USA, 2012.

5. Birnbaum, H.K. Mechanisms of Hydrogen Related Fracture of Metals. In Environment Induced Cracking of Metals, Proceedings of the First International Conference on Environment-Induced Cracking of Metals; Gangloff, R.P., Ives, M.B. Eds.; NACE: Houston, TX, USA, 1990; pp. 21-29.

6. Duquette, D. Corrosion Fatigue Crack Initiation Processes: A State of the Art Review. In Environment Induced Cracking of Metals, Proceedings of the First International Conference on Environment-Induced Cracking of Metals; Gangloff, R.P., Ives, M.B. Eds.; NACE: Houston, TX, USA, 1990; pp. 45-53.

7. Sieradzki, K. Atomistic \& Microchemical Aspects of Environment Induced Cracking of Metals. In Environment Induced Cracking of Metals, Proceedings of the First International Conference on Environment-Induced Cracking of Metals; Gangloff, R.P., Ives, M.B. Eds.; NACE: Houston, TX, USA, 1990; pp. 125-137.

8. Tang, H.; Basaran, C.A. Damage Mechanics Based Fatigue Life Prediction Model for Solder Joints. Trans. ASME J. Electron. Packag. 2003, 125, 120-125.

9. Prigogine, I. Introduction to Thermodynamics of Irreversible Processes; Interscience: New York, NY, USA, 1961.

10. Onsager, L. Reciprocal Relations in Irreversible Processes. J. Phys. 1931, 37, 2265-2279.

11. de Groot, S.R.; Mazur, P. Non-Equilibrium Thermodynamics; Wiley: New York, NY, USA, 1962.

12. Martyushev, L.M.; Seleznev, V.D. Maximum Entropy Production Principle in Physics. J. Chem. Biol. 2006, 426, 1-45.

13. Weertman, J. Elementary Dislocation Theory; Oxford University Press: New York, NY, USA, 1992.

14. Gutman, E. Mechanochemistry of Materials; Cambridge International Science Publishing: Cambridge, UK, 1998.

15. Lu, B.; Luo, J. Synergism of Electrochemical and Mechanical Factors in Erosion-Corrosion. J. Phys. Chem. 2006, 110, 4217-4231.

16. Amiri, M.; Khonsari, M. On the Thermodynamics of Friction and Wear-A Review. Entropy 2010, 12, 1021-1049.

17. Abdel-Aal, H. On the Interdependence between Kinetics of Friction-Released Thermal Energy and the Transition in Wear Mechanisms During Sliding of Metallic Pairs. J. Wear 2003, 254, 884-900.

18. Klamecki, B.E. An Entropy Based Model of Plastic Deformation Energy Dissipation in Sliding. J. Wear 1984, 96, 319-329.

19. Doelling, K.L.; Ling, F.F.; Bryant, M.D.; Heilman, B.P. An Experimental Study of the Correlation between Wear and Entropy Flow in Machinery Components. J. Appl. Phys. 2000, 88, 2999-3003.

20. Maugis, D. Contact, Adhesion, and Rupture of Elastic Solids; Springer: Berlin, Germany, 2000.

21. Naderi, M.; Amiri, M.; Khonsari, M. On the Thermodynamic Entropy of Fatigue Fracture. Process. R. Soc. 2010, 466, 423-438.

22. Leff, H.S. Removing the Mystery of Entropy and Thermodynamics-Part V. J. The Physics Teacher, 2012, 50, 274-276. 
23. Basaran, C.; Yan, C.A. Thermodynamic Framework for Damage Mechanics of Solder Joints. J. Electron. Packag. Trans. ASME 1998, 120, 379-384.

24. Ontiveros, V.L. Strain Energy and Thermodynamic Entropy as Prognastic Measure of Crack Initiation in Aluminum. Ph.D. Dissertation, Uiversity of Maryland, Maryland, USA, 2013.

25. Amiri, M.; Khonsari, M. On the Role of Entropy Generation in Processes Involving Fatigue. Entropy 2012, 14, 24-31.

26. Whaley, P.W.; Chen, P.; Smith, G.M. Continuous Measurement of Material Damping During Fatigue Tests. J. Exp. Mech. 1984, 24, 342-348.

27. Uhlig, H.; Revie, R. Corrosion and Corrosion Control, 3rd ed.; Wiley: New York, NY, USA, 1985.

28. Li, S.; Basaran, C.A. Computational Damage Mechanics Model for Thermomigration. J. Mech. Mater. 2009, 41, 271-278.

29. Nosonovsky, M.; Bhushan, B. Thermodynamics of Surface Degradation, Self-Organization, and Self-Healing for Biomimetic Surfaces. Philos. Trans. 2009, 367, 1607-1627.

30. Abdel-Aal, H. On the Role of Intrinsic Material Response in Failure of Tribo Systems. J. Wear 2005, 259, 1372-1381.

31. DuQuesnay, D.L.; Underhill, P.R.; Britt, H.J. Fatigue Crack Growth from Corrosion Fatigue in 7075-T6511 Aluminum Alloy under Aircraft Loading. Int. J. Fatigue, 2003, 25, 371-377.

32. Hoeppner, D.W. Parameters that Input to Application of Damage Tolerance Concepts to Critical Engine Components. In Proceedings of the AGARD Conference, San Antonio, TX, USA, April 1985.

33. Arriscorreta, C.A. Statistical Modeling for the Corrosion Fatigue of Aluminum Alloys 7075-T6 and 2024-T3. Ph.D. Dissertation, University of Utah, Utah, USA, 2012.

34. Kondo, Y. Prediction of Fatigue Crack Initiation Life Based on Pit Growth. J. Corros. Sci. 1989, $45,7-11$.

35. Lindley, T.C.; McIntyre, P.; Trant, P.J. Fatigue Crack Initiation at Corrosion Pits. J. Metals Technol. 1982, 9, 135-142.

36. Hoeppner, D.W. Model for Prediction of Fatigue Lives Based Upon a Pitting Corrosion Fatigue Process. In Fatigue Mechanisms; Proceeding of an ASTM NBS-NSF Symposium, Kansas City, MO, USA, 22-24 May 1978; Fong, J.T., Ed.; American Society for Testing and Materials: Philadelphia, USA, 1979; pp. 841-870.

37. Harlow, D.G.; Wei, R.P. Probability Approach for Prediction of Corrosion and Corrosion Fatigue Life. AIAA J. 1994, 32, 2073-2082.

38. Atkinson, J.D.; Yu, J.; Chen, Z.Y.; Zhao, Z.J. Modeling of Corrosion Fatigue Crack Growth Plateux for RPV Steels in High Temperature Water. J. Nucl. Eng. Des. 1998, 184, 13-25.

39. Hoeppner, D.W.; Chandrasekaran, V.; Taylor, A. Review of Pitting Corrosion Fatigue Model. In Proceedings of the 20th ICAF Symposium, Structural Integrity for the Next Millenium, Dayton, OH, USA, July, 2000.

40. Rong, W. A Fracture Model of Corrosion Fatigue Crack Propagation of Aluminum Alloys Based on the Material Elements Fracture Ahead of a Crack Tip. Int. J. Fatigue 2008, 30, 1376-1386. 
41. Chen, G.S.; Wan, K.-C.; Gao, M.; Wei, R.P.; Flournoy, T.H. Transition from Pitting to Fatigue Crack Growth-Modeling of Corrosion Fatigue Crack Nucleation in a 2024-T3 Aluminum Alloy. J. Mater. Sci. Eng. 1996, 219, 126-132.

42. Shi, P.; Mahadevan, S. Damage Tolerance Approach for Probabilistic Pitting Corrosion Fatigue Life Prediction. J. Eng. Fract. Mech. 2001, 68, 1493-1507.

43. Sankaran, K.; Perez, R.; Jata, K.V. Effects of Pitting Corrosion on the Fatigue Behavior of Aluminum Alloy 7075-T6: Modeling and Experimental Studies. J. Mater. Sci. Eng. 2001, 297, 223-229.

44. Gibbs, J.W. The Scientific Papers of J. Williard Gibbs; Ox Bow Press: Woodbridge, ON, Canada, 1993; Volume 1.

45. Ziegler, H. An Introduction to Thermomechanics; North-Holland: Amesterdam, The Netherlands, 1983.

46. Lemaitre, J.; Chaboche, J.L. Mechanics of Solid Materials; Cambridge University Press: Cambridge, United Kingdom, 2002.

47. Bridgman, P.W. The Nature of Thermodynamics; Harvard University Press: Cambridge, UK, 1941.

48. Kondepudi, D.; Prigogine, I. Modern Thermodynamics: From Heat Engines to Dissipative Structures; Wiley: New York, NY, USA, 1998.

49. Basaran, C. Implementation of a ThermodynamicFramework for Damage Mechanics of Solder Interconnects in Microelectronics Packaging. Int. J. Damage Mech. 2002, 11, 87-108.

50. Basaran, C.; Nie, S.A. Thermodynamics Based Damage Mechanics Model for Particulate Composites. Int. J. Solids Struct. 2007, 44, 1099-1114.

51. Basaran, C.; Nie, S. An Irreversible Thermodynamic Theory for Damage Mechanics of Solids. Int. J. Damage Mech. 2004, 13, 205-224.

52. Temfack, T.; Basaran, C. Experimental Verification of Thermodynamic Fatigue Life Prediction Model Using Entropy as Damage Metric. J. Mater. Sci. Technol. 2015, 31, 1627-1632.

53. Yan, C.Y. A Damage Mechanics Based General Purpose Interface Contact Element, Ph.D. Dissertation, State University of New York at Buffalo, Buffalo, New York, USA, 1997.

54. Tucker, J.P.; Chan, D.D.K.; Subbarayan, G.G.; Handwerker, C.A. Maximum Entropy Fracture Model and Fatigue Fracture of Mixed $\mathrm{SnPb} / \mathrm{Sn}_{3.0} \mathrm{Ag}_{0.5} \mathrm{Cu}$ Solder Alloys. In Proceedings of the 13th IEEE Intersociety Conference on Thermal and Thermomechanical Phenomena in Electronic Systems, San Diego, CA, USA, 30 May-1 June 2012; pp. 870-879.

55. Chan, D.; Subbarrayan, G.; Nguyen, L. Maximum-Entropy Principle for Modeling Damage and Fracture in Solder Joints. J. Electron. Mater. 2012, 41, 398-411.

56. Shannon, C.E. A Mathematical Theory of Communication. J. Bell Syst. Technol. 1948, 27, 379-423.

57. Bowley, R.; Sanchez, M. Introductory Statistical Mechanics, 2nd ed.; Oxford University Press: Oxford, UK, 1999.

58. Gangloff, R.P. Environmental Cracking Corrosion Fatigue; In Corrosion Tests and Standards: Application and Interpretation, 2nd ed.; Baboian, R., Ed.; ASTM International: West Conshohocken, PA, USA, 2005; pp. 302-321.

59. Jones, D.A. Principles and Prevention of Corrosion, 2nd ed.; Upper Saddle River: Upper Hall, NJ, USA, 1996. 
60. Seller, M.S.; Schultz, A.J.; Basaran, C.; Kofke, D.A. bSn Grain Boundary Structure and Self-diffusivity via Molecular Dynamics Simulation. J. Phys. Rev. B 2010, 81, 134111.

61. Kamoutsi, H.; Haidemenopoulos, G.; Bontozoglou, V.; Pantelakis, S. Corrosion-induced Hydrogen Embrittlement in Aluminum Alloy 2024. Corros. Sci. 2006, 48, 1209-1224.

62. Takano, N. Hydrogen Diffusion and Embrittlement in 7075 Aluminum Alloy. Mater. Sci. Eng. A 2008, 483-484, 36-339.

63. El-Amoush, A.S. An Investigation of Hydrogen-induced Hardening in 7075-T6 Aluminum Alloy. J. Alloys Compd. 2008, 465, 497-501.

64. Qiao, L.J.; Mao, S.X. Thermodynamic Analysis on the Role of Hydrogen in Anodic Stress Corrosion Cracking. J. Acta Metal Mater. 1995, 43, 3989-4001.

65. Mao, S.X.; Li, M. Mechanics and Thermodynamics on the Stress and Hydrogen Interaction in Crack Tip Stress Corrosion: Experiment and Theory. J. Mech. Phys. Solids 1998, 46, 1125-1137.

66. Gu, B.; Luo, J.; Mao, X. Hydrogen-Facilitated Anodic Dissolution-Type Stress Corrosion Cracking of Pipeline Steels in Near-Neutral pH Solution. Corrosion 1999, 55, 96-106.

67. Lu, B.T.; Luo, J.; Norton, P.; Ma, H. Effects of Dissolved Hydrogen and Elastic and Plastic Deformation on Active Dissolution of Pipeline Steel in Anaerobic Groundwater of Near-Neutral pH. Acta Mater. 2009, 57, 41-49.

68. Qiao, L.J.; Luo, J.L.; Mao, X. The Role of Hydrogen in the Process of Stress Corrosion Cracking of Pipeline Steels in Dilute Carbonate-Bicarbonate Solution. J. Mater. Sci. Lett. 1997, 16, 516-520.

69. Hu, X.; Huang, Z.; Qiao, L.; Chu, W. Effect of Hydrogen and Stress on Anodic Dissolution. J. Chin. Soc. Corros. Prot. 1996, 16, 187.

70. Sudarshan, T.S.; Louthan, M.R. Gaseous Environment Effect on Fatigue Behaviour of Metals. Int. Mater. Rev. 1987, 32, 121-151.

71. Grinberg, N. The Effect of Vacuum on Fatigue Crack Growth. Int. J. Fatigue 1982, 4, 83-95.

72. Liu, J.J.; Sun, J.J.; Hu, H.Y.; Xing, X.S. The Life Prediction for Materials under the Corrosion of Seawater. Acta Phys. Sin. 2005, 54, 2414-2417.

73. Gao, M.; Pao, P.S.; Wei, R.P. Chemical and Metallurgical Aspects of Environmentally Assisted Fatigue Crack Growth in 7075-T651 Aluminum Alloy. J. Metall. Mater. Trans. A 1988, 19, 1739-1750.

74. Griffith, A.A. The Phenomena of Rupture and Flow in Solids. Trans. R. Soc. 1921, 221, 163-198.

75. Huang, X.G.; Xu, J.Q.; Feng, M.L. Energy Principle of Corrosion Environment Accelerating Crack Propagation During Anodic Dissolution Corrosion Fatigue. J. Shanghai Jiaotong Univ. (Sci.) 2013, 18, 190-196.

76. Chun, Y.G.; Pyun, S.I. Crack Closure in the Fatigue Crack Propagation of a Cu-2wt.\%Be Alloy in Dry Air and Ammoniacal Solution. Mater. Sci. Eng. 1996, 206, 49-54.

77. Romaniv, O.R.; Nikiforchin, G.N.; Andrusiv, N.N. The Effect of Cracks Closure and the Estimation of Cyclic Crack Resistance of Structural Alloys. J. Phys., Chem. Mech. Mater. 1983, 3, 47-61. 
78. Fontea, M.D.; Romeirob, F.; Freitasc, M.D. The Effect of Microstructure and Environment on Fatigue Crack Propagation in 7049 Aluminium Alloy at Negative Stress Ratios. Int. J. Fatigue 2003, 25, 1209-1216.

79. Gangloff, R.P. Crack Size Effects on the Chemical Driving Force for Aqueous Corrosion Fatigue. Metall. Mater. Trans. 1985, 16, 953-969.

80. Bruck, H.A.; McNeill, S.R.; Sutton, M.A.; Iii, W.H.P. Digital Image Correlation Using Newton-Raphson Method of Partial Differential Correction. Exp. Mech. 1989, 29, 261-267.

81. Alexopoulos, N.D.; Papanikos, P. Experimental and Theoretical Studies of Corrosion-Induced Mechanical Properties Degradation of Aircraft 2024 Aluminum Alloy. J. Mater. Sci. Eng. 2008, 498, 248-257.

82. Smetanin, M.; Denga, Q.; Weissmu, J.R. Dynamic Electro-Chemo-Mechanical Analysis During Cyclic Voltammetry. J. Phys. Chem. 2011, 13, 17313-17322.

83. Valincius, G. Electrocapillary Equations of Solid Electrodes. J. Electroanal. Chem. 1999, 478, 40-49.

84. Sahal, M.; Creus, J.; Sabot, R.; Feaugas, X. The Effects of Dislocation Patterns on the Dissolution Process of Polycrystalline Nickel. J. Acta Mater. 2006, 54, 2157-2167.

85. Ieong, M.; Doris, B.; Kedzierski, J.; Rim, K.; Yang, M. Silicon Device Scaling to the Sub-10-nm Regime. J. Sci. 2004, 306, 2057-2060.

86. Choi, Y.S.; Numata, T.; Nishida, T.; Harris, R.; Thompson, S.E. Impact of Mechanical Stress on Gate Tunneling Currents of Germanium Silicon P-Type Metal-Oxide-Semiconductor Field-Effect Transistors and Metal Gate Work Function. J. Appl. Phys. 2008, 103, 064510.

87. Larché, F.; Cahn, J.W. The Effect of Self-Stress on Diffusion in Solids. J. Acta Met. 1982, 30, 1835-1845.

88. Larché, F.C.; Cahn, J.W. The Interactions of Composition and Stress in Crystalline Solids. J. Acta Met. 1985, 33, 331-357.

89. Stashchuk, M.H. Stress-Induced Cathodic and Anodic Regions on the Boundary between an Elliptic Hole and a Medium. J. Mater. Sci. 2011, 46, 36-54.

90. Xiao, J.; Chaudhuri, S. Predictive Modeling of Localized Corrosion: An Application to Aluminum Alloys. J. Electrochimica Acta 2011, 56, 5630-5641.

91. Birbilis, N.; Buchheit, R.G. Electrochemical Characteristics of Intermetallic Phases in Aluminum Alloys An Experimental Survey and Discussion. J. Electrochem. Soc. 2005, 152, 140-151.

92. Pao, P.; Feng, C.; Gill, S. Corrosion Fatigue Crack Initiation in Aluminum Alloys 7075 and 7050. J. Corros. 2000, 10, 1022-1032.

93. Costa-Matto, H.D.; Pacheco, P. Non-Isothermal Low-Cycle Fatigue Analysis of ElastoViscoplastic Materials. Mech. Res. Commun. 2009, 36, 428-436.

94. Manson, S.S. Thermal Stress and Low Cycle Fatigue; McGraw-Hill: New York, NY, USA, 1996.

95. Whaley, P.W. A Thermodynamic Approach to Metal Fatigue. In Proceedings of the ASME International Conference in Advances in Life Prediction Methods, Albany, NY, USA, April, 1983.

96. Norris, K.; Landzberg, A. Reliability of Controlled Collapse Interconnections. IBM J. Res. Dev. 1969, 13, 266-277.

97. Bayerer, R.; Tobias, H.; Thomas, L.; Lutz, J.; Feller, M. Model for Power Cycling Lifetime of IGBT Modules-Various Factors Influencing Lifetime. In Proceedings of the 2008 5th 
International Conference on Integerated Power Electronics Systems, Nuremberg, Germany, 11-13 March 2008; pp. 1-6.

98. Harlow, D.G.; Wei, R.P. A Probability Model for the Growth of Corrosion Pits in Aluminum Alloys Induced by Constituent Particles. J. Eng. Fract. Mech. 1998, 59, 305-325.

99. Byington, C.; Watson, M.; Edwards, D. Data-Driven Neural Network Methodology to Remaining Life Predictions for Aircraft Actuator Components. In Proceedings of the IEEE Aerospace Conference, New York, NY, USA, 6-13 March 2004; pp. 3581-3589.

100. Schwabacher, M.; Goebel, K. A Survey of Artificial Intelligence for Prognostics. In AAAI Fall Symposium, Arlington, VA, USA, 8-11 November 2007.

101. Bhangu, B.S.; Bentley, P.; Stone, D.A.; Bingham, C.M. Nonlinear Observers for Predicting State-of-Charge and State-of-Health of Lead-Acid Batteries for Hybrid-Electric Vehicles. IEEE Trans. Veh. Technol. 2005, 54, 783-798.

102. Imanian, A.; Modarres, M. A Thermodynamic Entropy Based Approach for Prognosis and Health Management. In Prognostics and Health Management Society Conference, Fort Worth, TX, USA, 29 September-2 October 2014.

103. Nystad, B.H. Technical Condition Indexes and Remaining Useful Life of Aggregated Systems. Ph.D. Dissertation, Norwegian University of Science and Technology, Trondheim, Norway, 2008.

104. Noortwijk, J.M.V. A Survey of the Application of Gamma Processes in Maintenance. Reliab. Eng. Syst. Saf. 2009, 94, 2-21.

(C) 2015 by the authors; licensee MDPI, Basel, Switzerland. This article is an open access article distributed under the terms and conditions of the Creative Commons Attribution license (http://creativecommons.org/licenses/by/4.0/). 